\title{
On the Sum of Generalized Hukuhara Differentiable Fuzzy Functions
}

\author{
Yurilev Chalco-Cano ${ }^{1(\bowtie)}\left(\mathbb{D}\right.$, A. Khastan ${ }^{2}\left(\mathbb{D}\right.$, and Antonio Rufián-Lizana ${ }^{3}$ \\ 1 Departamento de Matemática, Universidad de Tarapacá, Casilla 7D, Arica, Chile \\ ychalco@uta.cl \\ 2 Department of Mathematics, Institute for Advanced Studies in Basic Sciences, \\ Zanjan, Iran \\ khastan@gmail.com \\ 3 Departamento de Estadística e Investigación Operativa, Universidad de Sevilla, \\ Sevilla, Spain
}

\begin{abstract}
In this article we present new results on the sum of $g H$ differentiable fuzzy functions. We give conditions so that the sum of two $g H$-differentiable fuzzy functions become $g H$-differentiable. We present also practical rules for obtaining the $g H$-derivative of the sum of fuzzy functions.
\end{abstract}

Keywords: Fuzzy functions $\cdot$ gH-differentiable fuzzy functions · Algebra of gH-differentiable fuzzy functions

\section{Introduction}

The generalized Hukuhara differentiability ( $g H$-differentiability) for fuzzy functions is a very useful concept in the area of fuzzy mathematical analysis. This concept has been very important in the development of various topics into fuzzy theory, for instance, fuzzy differential equations and fuzzy optimization problems.

Obtaining the $g H$-derivative of an interval-valued function directly from the definition is a rather complex task. In contrast, the use of the (lateral) differentiability of its endpoint functions considerably simplifies the problem. In this direction, a characterization of the $g H$-differentiable fuzzy functions through of the (lateral) differentiability of its endpoint functions was obtained in [4].

Calculus for fuzzy functions is an important topic. Several properties of the $g H$-differentiable fuzzy functions have been obtained in [2-5]. In particular, some results on the algebra of $g H$-differentiable fuzzy functions have been presented in $[1,3,5]$.

This article was partially supported by the project UTA-Mayor Number 4745-19. 
In this paper we present some results on the sum of $g H$-differentiable fuzzy functions. We prove that the $(k) g H$-derivative is a linear operator. We show also that the sum of two $(k) g H$-differentiable fuzzy functions with different $k \in$ $\{i, i i, i i i, i v\}$ is not always $g H$-differentiable. We give conditions so that sum of fuzzy functions become $g H$-differentiable.

\section{Preliminaries}

We denote by $\mathbb{I}$ the family of all bounded closed intervals in $\mathbb{R}$, i.e.,

$$
\mathbb{I}=\{A=[\underline{a}, \bar{a}] / \underline{a}, \bar{a} \in \mathbb{R} \text { and } \underline{a} \leq \bar{a}\} .
$$

On $\mathbb{I}$ we consider the algebraic operations "+" and "." by (see, e.g., [6])

$$
[\underline{a}, \bar{a}]+[\underline{b}, \bar{b}]=[\underline{a}+\underline{b}, \bar{a}+\bar{b}] \quad \lambda \cdot[\underline{a}, \bar{a}]=[\min \{\lambda \underline{a}, \lambda \bar{a}\}, \max \{\lambda \underline{a}, \lambda \bar{a}\}] .
$$

Note that $(\mathbb{I},+, \cdot)$ is a quasilinear space [7]. An interval $A=[\underline{a}, \bar{a}]$ with $\underline{a}<\bar{a}$ does not have an additive inverse. So, in the article [8] was introduced the generalized Hukuhara difference " $\ominus_{g H}$ " by

$$
[\underline{a}, \bar{a}] \ominus_{g H}[\underline{b}, \bar{b}]=[\min \{\underline{a}-\underline{b}, \bar{a}-\bar{b}\}, \max \{\underline{a}-\underline{b}, \bar{a}-\bar{b}\}] .
$$

The $g H$-difference is a complementary operation in the quasilinear space $(\mathbb{I},+, \cdot)$. We note that the $g H$-difference $A \ominus_{g H} B$, of two intervals $A$ and $B$, there is always.

Let $\mathbb{R}_{\mathcal{F}}$ denote the family of all fuzzy numbers. Let us denote by $[u]^{\alpha}=$ $\left[\underline{u}_{\alpha}, \bar{u}_{\alpha}\right] \in \mathbb{R}$ the $\alpha$-level set of $u$, for all $\alpha \in[0,1]$.

Let $u, v \in \mathbb{R}_{\mathcal{F}}$, with $\alpha$-levels represented by $\left[\underline{u}_{\alpha}, \bar{u}_{\alpha}\right]$ and $\left[\underline{v}_{\alpha}, \bar{v}_{\alpha}\right]$, respectively, and let $\lambda \in \mathbb{R}$. The addition $u+v$ and the scalar multiplication $\lambda u$ are defined via the $\alpha$-levels by

$$
\begin{gathered}
{[u+v]^{\alpha}=\left[(\underline{u+v})_{\alpha},(\overline{u+v})_{\alpha}\right]=\left[\underline{u}_{\alpha}+\underline{v}_{\alpha}, \bar{u}_{\alpha}+\bar{v}_{\alpha}\right],} \\
{[\lambda u]^{\alpha}=\left[(\underline{\lambda u})_{\alpha},(\overline{\lambda u})_{\alpha}\right]=\left[\min \left\{\lambda \underline{u}_{\alpha}, \lambda \bar{u}_{\alpha}\right\}, \max \left\{\lambda \underline{u}_{\alpha}, \lambda \bar{u}_{\alpha}\right\}\right],}
\end{gathered}
$$

for every $\alpha \in[0,1]$. In this case $\left(\mathbb{R}_{\mathcal{F}},+, \cdot\right)$ is a quasilinear space [7].

Definition 1. ([9]) Given two fuzzy numbers $u$, v, the generalized Hukuhara difference ( $g H$-difference for short) is the fuzzy interval $w$, if it exists, such that

$$
u \ominus_{g H} v=w \Leftrightarrow\left\{\begin{array}{l}
(i) u=v+w, \\
o r \quad(i i) v=u+(-1) w .
\end{array}\right.
$$

It may happen that there is not $g H$-difference of two fuzzy numbers [9].

If $u \ominus_{g H} v$ exists then, in terms of $\alpha$-levels, we have

$$
\begin{aligned}
{\left[u \ominus_{g H} v\right]^{\alpha} } & =[u]^{\alpha} \ominus_{g H}[u]^{\alpha} \\
& =\left[\min \left\{\underline{u}_{\alpha}-\underline{v}_{\alpha}, \bar{u}_{\alpha}-\bar{v}_{\alpha}\right\}, \max \left\{\underline{u}_{\alpha}-\underline{v}_{\alpha}, \bar{u}_{\alpha}-\bar{v}_{\alpha}\right\}\right],
\end{aligned}
$$

for all $\alpha \in[0,1]$, where $[u]^{\alpha} \ominus_{g H}[u]^{\alpha}$ denotes the gH-difference between two intervals.

Given $u, v \in \mathbb{R}_{\mathcal{F}}$ we define the distance between $u$ and $v$ by

$$
D(u, v)=\sup _{\alpha \in[0,1]} \max \left\{\left|\underline{u}_{\alpha}-\underline{v}_{\alpha}\right|,\left|\bar{u}_{\alpha}-\bar{v}_{\alpha}\right|\right\} .
$$




\section{Generalized Hukuhara Differentiable Fuzzy Functions}

Henceforth, $T=] a, b\left[\right.$ denotes an open interval in $\mathbb{R}$. A function $F: T \rightarrow \mathbb{R}_{\mathcal{F}}$ is said to be a fuzzy function. For each $\alpha \in[0,1]$, associated with $F$, we define the family of interval-valued functions $F_{\alpha}: T \rightarrow \mathbb{I}$ given by $F_{\alpha}(x)=[f(x)]^{\alpha}$. For any $\alpha \in[0,1]$, we denote

$$
F_{\alpha}(x)=\left[\underline{f}_{\alpha}(x), \bar{f}_{\alpha}(x)\right]
$$

Here, for each $\alpha \in[0,1]$, the endpoint functions $\underline{f}_{\alpha}, \bar{f}_{\alpha}: T \rightarrow \mathbb{R}$ are called upper and lower functions of $F$, respectively.

The following definition is the well-known concept of generalized Hukuhara differentiable fuzzy functions ( $g H$-differentiable fuzzy functions, for short) based on the $g H$-difference of fuzzy intervals.

Definition 2. ([3]) The $g H$-derivative of a fuzzy function $F: T \rightarrow \mathbb{R}_{\mathcal{F}}$ at $x_{0} \in T$ is defined as

$$
F^{\prime}\left(x_{0}\right)=\lim _{h \rightarrow 0} \frac{1}{h}\left[F\left(x_{0}+h\right) \ominus_{g H} F\left(x_{0}\right)\right] .
$$

If $F^{\prime}\left(x_{0}\right) \in \mathbb{R}_{\mathcal{F}}$ satisfying (2) exists, we say that $F$ is $g H$-differentiable at $x_{0}$.

Obtaining the $g H$-derivative of a fuzzy function via (2) is a rather complex problem. The following result characterizes the $g H$-differentiability of $F$ in terms of the differentiability of its endpoint functions $\underline{f}_{\alpha}$ and $\bar{f}_{\alpha}$ which makes it more practice to calculate the $g H$-derivative.

Theorem 1. ([4]) Let $F: T \rightarrow \mathbb{R}_{\mathcal{F}}$ be a fuzzy function and $x \in T$. Then $F$ is $g H$-differentiable at $x$ if and only if one of the following four cases holds:

(i) $\underline{f}_{\alpha}$ and $\bar{f}_{\alpha}$ are differentiable at $x$, uniformly in $\alpha \in[0,1],\left(\underline{f}_{\alpha}\right)^{\prime}(x)$ is monotonic increasing and $\left(\bar{f}_{\alpha}\right)^{\prime}(x)$ is monotonic decreasing as functions of $\alpha$ and $\left(\underline{f}_{1}\right)^{\prime}(x) \leq\left(\bar{f}_{1}\right)^{\prime}(x)$. In this case,

$$
F_{\alpha}^{\prime}(x)=\left[\left(\underline{f}_{\alpha}\right)^{\prime}(x),\left(\bar{f}_{\alpha}\right)^{\prime}(x)\right]
$$

for all $\alpha \in[0,1]$.

(ii) $\underline{f}_{\alpha}$ and $\bar{f}_{\alpha}$ are differentiable at $x$, uniformly in $\alpha \in[0,1],\left(\underline{f}_{\alpha}\right)^{\prime}(x)$ is monotonic decreasing and $\left(\bar{f}_{\alpha}\right)^{\prime}(x)$ is monotonic increasing as functions of $\alpha$ and $\left(\bar{f}_{1}\right)^{\prime}(x) \leq\left(\underline{f}_{1}\right)^{\prime}(x)$. In this case,

$$
F_{\alpha}^{\prime}(x)=\left[\left(\bar{f}_{\alpha}\right)^{\prime}(x),\left(\underline{f}_{\alpha}\right)^{\prime}(x)\right],
$$

for all $\alpha \in[0,1]$. 
(iii) $\left(\underline{f}_{\alpha}\right)_{+/-}^{\prime}(x)$ and $\left(\bar{f}_{\alpha}\right)_{+/-}^{\prime}(x)$ exist uniformly in $\alpha \in[0,1],\left(\underline{f}_{\alpha}\right)_{+}^{\prime}(x)=$ $\left(\bar{f}_{\alpha}\right)_{-}^{\prime}(x)$ is monotonic increasing and $\left(\bar{f}_{\alpha}\right)_{+}^{\prime}(x)=\left(\underline{f}_{\alpha}\right)_{-}^{\prime}(x)$ is monotonic decreasing as functions of $\alpha$ and $\left(\underline{f}_{1}\right)_{+}^{\prime}(x) \leq\left(\bar{f}_{1}\right)_{+}^{\prime}(x)$. In this case,

$$
F_{\alpha}^{\prime}(x)=\left[\left(\underline{f}_{\alpha}\right)_{+}^{\prime}(x),\left(\bar{f}_{\alpha}\right)_{+}^{\prime}(x)\right]=\left[\left(\bar{f}_{\alpha}\right)_{-}^{\prime}(x),\left(\underline{f}_{\alpha}\right)_{-}^{\prime}(x)\right],
$$

for all $\alpha \in[0,1]$.

(iv) $\left(\underline{f}_{\alpha}\right)_{+/-}^{\prime}(x)$ and $\left(\bar{f}_{\alpha}\right)_{+/-}^{\prime}(x)$ exist uniformly in $\alpha \in[0,1],\left(\underline{f}_{\alpha}\right)_{+}^{\prime}(x)=$ $\left(\bar{f}_{\alpha}\right)_{-}^{\prime}(x)$ is monotonic decreasing and $\left(\bar{f}_{\alpha}\right)_{+}^{\prime}(x)=\left(\underline{f}_{\alpha}\right)_{-}^{\prime}(x)$ is monotonic increasing as functions of $\alpha$ and $\left(\bar{f}_{1}\right)_{+}^{\prime}(x) \leq\left(\underline{f}_{-}\right)_{+}^{\prime}(x)$. In this case,

$$
F_{\alpha}^{\prime}(x)=\left[\left(\bar{f}_{\alpha}\right)_{+}^{\prime}(x),\left(\underline{f}_{\alpha}\right)_{+}^{\prime}(x)\right]=\left[\left(\underline{f}_{\alpha}\right)_{-}^{\prime}(x),\left(\bar{f}_{\alpha}\right)_{-}^{\prime}(x)\right],
$$

for all $\alpha \in[0,1]$.

From Theorem 1 we can distinguish four cases. We say that an interval-valued function $F: T \rightarrow \mathbb{R}_{\mathcal{F}}$ is $(k) g H$-differentiable if case $k$ in Theorem 1 holds, for $k \in\{i, i i, i i i, i v\}$. Note that if $F: T \rightarrow \mathbb{R}_{\mathcal{F}}$ is $g H$-differentiable at $x_{0}$ in more than one case then $F$ is $g H$-differentiable at $x_{0}$ in all four cases and $F^{\prime}\left(x_{0}\right)$ is a trivial fuzzy number or singleton, i.e. $F^{\prime}\left(x_{0}\right)=\chi_{\{a\}}$ for some $a \in \mathbb{R}$.

\section{The Sum of $\boldsymbol{g H}$-differentiable Fuzzy Functions}

Given two fuzzy functions $F, G: T \rightarrow \mathbb{R}_{\mathcal{F}}$ the sum operation is defined by

$$
(F+G)(x)=F(x)+G(x),
$$

for all $x \in T$. Via the $\alpha$-levels, with $F_{\alpha}(x)=\left[\underline{f}_{\alpha}(x), \bar{f}_{\alpha}(x)\right]$ and $G_{\alpha}(x)=$ $\left[\underline{g}_{\alpha}(x), \bar{g}_{\alpha}(x)\right]$, for all $\alpha \in[0,1]$, we have

$$
\left(F_{\alpha}+G_{\alpha}\right)(x)=F_{\alpha}(x)+G_{\alpha}(x)=\left[\underline{f}_{\alpha}(x)+\underline{g}_{\alpha}(x), \bar{f}_{\alpha}(x)+\bar{g}_{\alpha}(x)\right],
$$

for all $x \in T$.

In this Section we present results on the $g H$-differentiability of $F+G$. We give rules for calculating it as well.

We start by showing that if $F$ and $G$ are $g H$-differentiable at $x_{0}$ with same type of $g H$-differentiability, then $F+G$ is also $g H$-differentiable at $x_{0}$ with the same type of $g H$-differentiability as $F$ and $G$.

Theorem 2. Let $F, G: T \rightarrow \mathbb{R}_{\mathcal{F}}$ be two fuzzy functions. If $F$ and $G$ are (k)gH-differentiable at $x_{0}$ for the same $k \in\{i, i i, i i i, i v\}$ then $F+G$ is $(k) g H$ differentiable at $x_{0}$. Moreover,

$$
(F+G)^{\prime}\left(x_{0}\right)=F^{\prime}\left(x_{0}\right)+G^{\prime}\left(x_{0}\right) .
$$


Proof. To prove the result we will consider separately the four possible cases for $k$. For $k \in\{1,2\}$, the results were proven in [3]. For case $k=i i i$, we consider $F_{\alpha}(x)=\left[\underline{f}_{\alpha}(x), \bar{f}_{\alpha}(x)\right]$ and $G_{\alpha}(x)=\left[\underline{g}_{\alpha}(x), \bar{g}_{\alpha}(x)\right]$, for all $x \in T$, and assume that $F$ and $G$ are (iii) $g H$-differentiable fuzzy functions at $x_{0}$ then, from Theorem 1 and properties of lateral derivatives, the lateral derivatives $\left(\underline{f}_{\alpha}+\underline{g}_{\alpha}\right)_{+/-}^{\prime}\left(x_{0}\right)$ and $\left(\bar{f}_{\alpha}+\bar{g}_{\alpha}\right)_{+/-}^{\prime}\left(x_{0}\right)$ exist uniformly in $\alpha \in[0,1]$. In addition, $\left(\underline{f}_{\alpha}+\underline{g}_{\alpha}\right)_{+}^{\prime}\left(x_{0}\right)=\left(\bar{f}_{\alpha}+\bar{g}_{\alpha}\right)_{-}^{\prime}\left(x_{0}\right)$ is monotonic increasing and $\left(\bar{f}_{\alpha}+\bar{g}_{\alpha}\right)_{+}^{\prime}\left(x_{0}\right)=\left(\underline{f}_{\alpha}+\underline{g}_{\alpha}\right)_{-}^{\prime}\left(x_{0}\right)$ is monotonic decreasing as functions of $\alpha$ and $\left(f_{1}+g_{1}\right)_{+}^{\prime}\left(x_{0}\right) \leq\left(\overline{\bar{f}}_{1}+\bar{g}_{1}\right)_{+}^{\prime}\left(x_{0}\right)$. Thus, from Theorem 1 , part $(i i i), F+G$ is a (iii) $g \bar{H}$-differentiable fuzzy function at $x$. Moreover

$$
\begin{aligned}
{\left[(F+G)^{\prime}\left(x_{0}\right)\right]^{\alpha} } & =\left[\left(\underline{f}_{\alpha}+\underline{g}_{\alpha}\right)_{+}^{\prime}\left(x_{0}\right),\left(\bar{f}_{\alpha}+\bar{g}_{\alpha}\right)_{+}^{\prime}\left(x_{0}\right)\right] \\
& =\left[\left(\underline{f}_{\alpha}\right)_{+}^{\prime}\left(x_{0}\right)+\left(\underline{g}_{\alpha}\right)_{+}^{\prime}\left(x_{0}\right),\left(\bar{f}_{\alpha}\right)_{+}^{\prime}\left(x_{0}\right)+\left(\bar{g}_{\alpha}\right)_{+}^{\prime}\left(x_{0}\right)\right] \\
& =\left[\left(\underline{f}_{\alpha}\right)_{+}^{\prime}\left(x_{0}\right),\left(\bar{f}_{\alpha}\right)_{+}^{\prime}\left(x_{0}\right)\right]+\left[\left(\underline{g}_{\alpha}\right)_{+}^{\prime}\left(x_{0}\right),\left(\bar{g}_{\alpha}\right)_{+}^{\prime}\left(x_{0}\right)\right] \\
& =\left[F^{\prime}\left(x_{0}\right)\right]^{\alpha}+\left[G^{\prime}\left(x_{0}\right)\right]^{\alpha},
\end{aligned}
$$

for all $\alpha \in[0,1]$, and so

$$
(F+G)^{\prime}\left(x_{0}\right)=F^{\prime}\left(x_{0}\right)+G^{\prime}\left(x_{0}\right) .
$$

The proof for $k=i v$ is similar, so we omit it.

From Theorem 2 it follows that the $(k) g H$-derivative is an additive operator, for $k \in\{i, i i, i i i, i v\}$. Proving that the $g H$-derivative is a linear operator is our main objective. For this, given a fuzzy function $F: T \rightarrow \mathbb{R}_{\mathcal{F}}$ and a $\lambda \in \mathbb{R}$, we define the product $\lambda \cdot F$ by

$$
(\lambda \cdot F)(x)=\lambda \cdot F(x)
$$

for all $x \in T$.

Theorem 3. Let $F: T \rightarrow \mathbb{R}_{\mathcal{F}}$ be a fuzzy function and let $\lambda \in \mathbb{R}$. If $F$ is (k) $g H$-differentiable at $x_{0}$, for some $k \in\{i, i i, i i i, i v\}$, then $\lambda \cdot F$ is also $(k) g H$ differentiable at $x_{0}$ and

$$
(\lambda \cdot F)^{\prime}\left(x_{0}\right)=\lambda \cdot F^{\prime}\left(x_{0}\right) .
$$

Proof. We consider $\lambda \in \mathbb{R}$ and $x \in T$, then from (1) and (3) we have

$$
\begin{aligned}
{[(\lambda \cdot F)(x)]^{\alpha} } & =[\lambda \cdot F(x)]^{\alpha} \\
& =\lambda \cdot[F(x)]^{\alpha} \\
& =\lambda \cdot\left[\underline{f}_{\alpha}(x), \bar{f}_{\alpha}(x)\right] \\
& =\left\{\begin{array}{l}
{\left[\lambda \underline{f}_{\alpha}(x), \lambda \bar{f}_{\alpha}(x)\right] \text { if } \lambda \geq 0} \\
{\left[\lambda \bar{f}_{\alpha}(x), \lambda \underline{f}_{\alpha}(x)\right] \text { if } \lambda<0 .}
\end{array}\right.
\end{aligned}
$$


To prove the result we consider separately the four possible cases for $k$. We start considering $k=i$ and so, from Theorem 1, we have that the endpoint functions $\underline{f}_{\alpha}$ and $\bar{f}_{\alpha}$ are differentiable at $x_{0}$, uniformly in $\alpha \in[0,1]$, and satisfies the monotonicity conditions in relation to $\alpha$ as in item (i) of the Theorem 1 . Now we consider two cases for $\lambda: \lambda \geq 0$ and $\lambda<0$.

If $\lambda \geq 0$, taking into account item (i) of Theorem 1 and properties of monotonous functions, we have that $\lambda \underline{f}_{\alpha}^{\prime}(x)$ is monotonic increasing and $\lambda \bar{f}_{\alpha}^{\prime}(x)$ is monotonic decreasing as functions of $\alpha$ and $\lambda \underline{f}_{1}^{\prime}(x) \leq \lambda \bar{f}_{1}^{\prime}(x)$. Then, from Theorem $1, \lambda F$ is an $(i) g H$-differentiable fuzzy function.

On other hand, if $\lambda<0$, taking into account item (i) of Theorem 1 and properties of monotonous functions, we have that $\lambda \bar{f}_{\alpha}^{\prime}(x)$ is monotonic increasing and $\lambda \underline{f}_{\alpha}^{\prime}(x)$ is monotonic decreasing as functions of $\alpha$ and $\lambda \bar{f}_{1}^{\prime}(x) \leq \lambda \underline{f}_{1}^{\prime}(x)$. Then, from Theorem $1, \lambda F$ is an $(i) g H$-differentiable fuzzy function.

In the same way we proof the cases for $k=i i, i i i, i v$.

Summarizing, from Theorem 2 and Theorem 3 we have our main result.

Corollary 1. The $(k) g H$-derivative is a linear operator, for each $k \in$ $\{i, i i, i i i, i v\}$.

Remark 1. Theorem 3 and Corollary 1 correct Remark 31 in [3], where the authors assert that the $(i) g H$-derivative and $(i i) g H$-derivative are not linear in general.

Following with our study, what happen with the $g H$-differentiability of the sum of two $(k) g H$-differentiable fuzzy functions for different $k$ ?. In general, $F+$ $G$ is not necessarily a $g H$-differentiable fuzzy function being $F$ and $G(k) g H$ differentiable fuzzy functions with different $k$. In fact, Example 1 in [5], $F+G$ is not $g H$-differentiable being that $F$ is $(i i) g H$-differentiable and $G$ is $(i v) g H$ differentiable.

In the same way, if $F$ is $(i) g H$-differentiable and $G$ is $(i i) g H$-differentiable then $F+G$ is $g H$-differentiable, when $F$ and $G$ are interval-valued functions which are particular cases of fuzzy functions, (see [5]). More precisely.

Theorem 4. ([5]) Let $F, G: T \rightarrow \mathbb{I}$ be two interval-valued functions. If $F$ is (i)gH-differentiable at $x_{0}$ and $G$ is $\left(\right.$ ii)gH-differentiable at $x_{0}$ then $F+G$ is either $(i) g H$-differentiable or $($ ii $) g H$-differentiable at $x_{0}$. Moreover, we have

$$
(F+G)^{\prime}\left(x_{0}\right)=F^{\prime}\left(x_{0}\right) \ominus_{g H}(-1) G^{\prime}\left(x_{0}\right) .
$$

However, in general Theorem 4 is not valid for fuzzy functions as we will show in the following example.

Example 1. We consider the fuzzy functions $F, G:(0,1) \rightarrow \mathbb{R}_{\mathcal{F}}$ defined via $\alpha$-levels by

$$
F_{\alpha}(x)=[\alpha, 2-\alpha] \cdot x^{2} \quad \text { and } \quad G_{\alpha}(x)=\left[0,1-\alpha^{2}\right] \cdot(1-x) .
$$


The endpoint functions are

$$
\begin{gathered}
\underline{f}_{\alpha}(x)=\alpha x^{2}, \quad \bar{f}_{\alpha}(x)=(2-\alpha) x^{2}, \\
\underline{g}_{\alpha}(x)=0, \quad \bar{g}_{\alpha}(x)=\left(1-\alpha^{2}\right)(1-x) ;
\end{gathered}
$$

which are differentiable. Also, we have that $F$ is $(i) g H$-differentiable and $G$ is (ii) $g H$-differentiable. Now, the endpoint functions of the fuzzy function $F+G$ are

$$
(\underline{F+G})_{\alpha}(x)=\underline{f}_{\alpha}(x)+\underline{g}_{\alpha}(x)=\alpha x^{2}
$$

and

$$
(\overline{F+G})_{\alpha}(x)=\bar{f}_{\alpha}(x)+\bar{g}_{\alpha}(x)=(2-\alpha) x^{2}+\left(1-\alpha^{2}\right)(1-x) .
$$

Clearly, the endpoint functions are differentiable and

$$
(\underline{F+G})_{\alpha}^{\prime}(x)=2 \alpha x
$$

and

$$
(\overline{F+G})_{\alpha}^{\prime}(x)=2(2-\alpha) x-\left(1-\alpha^{2}\right)=4 x-2 \alpha x-1+\alpha^{2} .
$$

Thus, the endpoint functions $(\underline{F+G})_{\alpha}$ and $(\overline{F+G})_{\alpha}$ do not satisfy any of the conditions of the Theorem 1 . Therefore $F+G$ is not $g H$-differentiable.

From (4) we can see that for obtaining $(F+G)^{\prime}(x)$ depends on the $g H$ difference. In fact, in Example 1 there is not the $g H$-difference $F^{\prime}\left(x_{0}\right) \ominus_{g H}$ $(-1) G^{\prime}\left(x_{0}\right)$. The $g H$-difference of two fuzzy numbers does not always exists while the $g H$-difference between interval always exists [9]. So we have to include conditions on the existence of the $g H$-difference such as in the following result.

Theorem 5. Let $F, G: T \rightarrow \mathbb{R}_{\mathcal{F}}$ be fuzzy functions. If $F$ is $(i) g H$-differentiable at $x_{0}$ and $G$ is (ii)gH-differentiable at $x_{0}$ and the $g H$-difference $F^{\prime}\left(x_{0}\right) \ominus_{g H}$ $(-1) G^{\prime}\left(x_{0}\right)$ exists, then $F+G$ is $g H$-differentiable and

$$
(F+G)^{\prime}\left(x_{0}\right)=F^{\prime}\left(x_{0}\right) \ominus_{g H}(-1) G^{\prime}\left(x_{0}\right) .
$$

Proof. Since the $g H$-difference $F^{\prime}\left(x_{0}\right) \ominus_{g H}(-1) G^{\prime}\left(x_{0}\right)$ exists, then one of the following cases holds:

$$
\begin{gathered}
\text { Case }(1)\left\{\begin{array}{l}
\operatorname{len}\left(F^{\prime}\left(x_{0}\right)\right) \geq \operatorname{len}\left(G^{\prime}\left(x_{0}\right)\right), \forall \alpha \in[0,1], \\
f^{\prime}\left(x_{0}\right)+g_{\alpha}^{\prime}\left(x_{0}\right) \text { is monotonic increasing respect to } \alpha \text { and } \\
\bar{f}_{\alpha}^{\prime}\left(x_{0}\right)+\bar{g}_{\alpha}^{\prime}\left(x_{0}\right) \text { is monotonic decreasing respect to } \alpha .
\end{array}\right. \\
\text { Case }(2)\left\{\begin{array}{l}
\text { len }\left(F^{\prime}\left(x_{0}\right)\right) \leq l e n\left(G^{\prime}\left(x_{0}\right)\right), \forall \alpha \in[0,1], \\
f_{\alpha}^{\prime}\left(x_{0}\right)+\underline{g}_{\alpha}^{\prime}\left(x_{0}\right) \text { is monotonic decreasing respect to } \alpha \text { and } \\
\overline{\bar{f}}_{\alpha}^{\prime}\left(x_{0}\right)+\bar{g}_{\alpha}^{\prime}\left(x_{0}\right) \text { is monotonic increasing respect to } \alpha .
\end{array}\right.
\end{gathered}
$$

If Case (1) holds then, from Theorem 1 item (i), $F+G$ is $(i) g H$-differentiable at $x_{0}$ and (5) holds. If Case (2) holds then, from Theorem 1 item (ii), $F+G$ is ( $i i) g H$-differentiable at $x_{0}$ and (5) holds. Therefore, $F+G$ is $(i) g H$ or $(i i) g H$ differentiable at $x_{0}$ and (5) holds.

We note that a similar result to Theorem 5 was obtained in [1] to case of strongly generalized differentiable fuzzy functions. 


\section{Conclusions}

In this article we have made a study on the sum of generalized Hukuhara differentiable fuzzy functions. The results obtained in this study should be very useful in fuzzy and interval optimization, fuzzy and interval differential equations and other topics in fuzzy and interval mathematical analysis.

\section{References}

1. Alikhani, R., Bahrami, F., Parvizi, S.: Differential calculus of fuzzy multi-variable functions and its applications to fuzzy partial differential equations. Fuzzy Sets Syst. 375, 100-120 (2019)

2. Armand, A., Allahviranloo, T., Gouyandeh, Z.: Some fundamental results on fuzzy calculus. Iran. J. Fuzzy Syst. 15, 27-46 (2018)

3. Bede, B., Stefanini, L.: Generalized differentiability of fuzzy-valued functions. Fuzzy Sets Syst. 230, 119-141 (2013)

4. Chalco-Cano, Y., Rodríguez-López, R., Jiménez-Gamero, M.D.: Characterizations of generalized differentiable fuzzy functions. Fuzzy Sets Syst. 295, 37-56 (2016)

5. Chalco-Cano, Y., Maqui-Huamán, G.G., Silva, G.N., Jiménez-Gamero, M.D.: Algebra of generalized Hukuhara differentiable interval-valued functions: review and new properties. Fuzzy Sets Syst. 375, 53-69 (2019)

6. Moore, R.E.: Methods and Applications of Interval Analysis. Studies in Applied and Numerical Mathematics. Society for Industrial and Applied Mathematics, Philadelphia (1979)

7. Rojas-Medar, M.A., Jiménez-Gamero, M.D., Chalco-Cano, Y., Viera-Brandão, A.J.: Fuzzy quasilinear spaces and applications. Fuzzy Sets Syst. 152, 173-190 (2005)

8. Stefanini, L., Bede, B.: Generalized Hukuhara differentiability of interval-valued functions and interval differential equations. Nonlinear Anal. 71(34), 1311-1328 (2009)

9. Stefanini, L.: A generalization of Hukuhara difference and division for interval and fuzzy arithmetic. Fuzzy Sets Syst. 161, 1564-1584 (2010) 